\title{
Whole-brain morphometric studies in alcohol addicts by voxel- based morphometry
}

\author{
Jinfeng $\mathrm{Li}^{1}$, Yonghao Wang ${ }^{2}$, Zhengyang $\mathrm{Xu}^{1}$, Tiefang $\mathrm{Liu}^{1}$, Xiao Zang ${ }^{1}$, Meng $\mathrm{Li}^{1}, \mathrm{Lin}^{\mathrm{Ma}}{ }^{1}$ \\ ${ }^{1}$ Department of Radiology, The First Medical Center of PLA General Hospital, Beijing 100853, China; ${ }^{2}$ Department of Ultrasound, The Eighth \\ Medical Center of PLA General Hospital, Beijing 100091, China \\ Contributions: (I) Conception and design: J Li; (II) Administrative support: L Ma; (III) Provision of study materials or patients: Y Wang; (IV) \\ Collection and assembly of data: Z Xu, X Zang ; (V) Data analysis and interpretation: T Liu, M Li; (VI) Manuscript writing: All authors; (VII) Final \\ approval of manuscript: All authors. \\ Correspondence to: Lin Ma. Department of Radiology, The First Medical Center of PLA General Hospital, Beijing 100853, China. \\ Email: cjrmalin@163.com.
}

\begin{abstract}
Background: To investigate the morphological changes of the whole-brain in patients with alcohol addiction using voxel-based morphometry (VBM).

Methods: Twenty alcohol addiction individuals and 20 healthy control volunteers, matched in gender, age, handedness and education, were enrolled as the alcohol addiction group and control group, respectively, from June 2017 to February 2018. The two groups underwent magnetic resonance imaging (MRI) scans of the whole-brain using a 3.0T MRI system, and the MRI images were analyzed by VBM. Total brain volume analysis was performed to observe the changes of local brain volume. Independent samples $t$-test was used for comparison of whole-brain volumes between the two groups.

Results: Gray matter volume (GMV) was significantly more reduced in the left middle frontal gyrus, left superior frontal gyrus, left precuneate gyrus, left gyrus rectus, right medial superior frontal gyrus, and right orbital gyrus in patients with alcohol addiction than in healthy controls. White matter volume (WMV) in the bilateral superior frontal gyrus was also significantly more reduced in patients with alcohol addiction than in healthy controls. Whole-brain VBM results showed that white matter and brain parenchyma volumes (BPVs) were significantly smaller in patients with alcohol addiction than in healthy controls (both $\mathrm{P}<0.05)$. There was no significant difference in GMV between patients with alcohol addiction and healthy controls $(\mathrm{P}>0.05)$. Conclusions: There are dominant areas of brain atrophy in patients with alcohol addiction. The VBM has a potential application value in detecting subtle brain atrophy in patients with alcohol addiction and providing an imaging basis in the diagnosis of alcohol addiction.
\end{abstract}

Keywords: Alcoholism; behaviors; addiction; magnetic resonance imaging (MRI); anthropometry; brain

Submitted Oct 12, 2019. Accepted for publication Oct 21, 2019.

doi: $10.21037 /$ atm.2019.10.90

View this article at: http://dx.doi.org/10.21037/atm.2019.10.90

\section{Introduction}

Voxel-based morphometry (VBM) is an objective automatic whole-brain analysis method that allows the detection of subtle morphometric inter-subject differences in brain structure (1-3). This technique has been widely used for measuring whole-brain volume in patients with some diseases including presenile dementia, amyotrophic lateral sclerosis, and optical neuromyelitis (4-8). Neuropathology evidence (9) exists indicating that alcoholic brain damage mainly occurs in the superior frontal gyrus, hypothalamus, and cerebellum. A neuroimaging study has demonstrated that magnetic resonance (MR) signal intensity of gray and white matter in the frontal lobe, insular lobe, and cerebellum decreased in patients with alcohol addiction (10). Xiao et al. (11) identified consistent regional gray matter 
(GM) atrophy particularly within several neurofunctional networks associated with alcohol dependence. Liu et al. (12) found a reduction of frontal lobe volume in elderly patients with chronic alcohol addiction in a semiquantitative analysis study. However, the particular morphological changes of the whole-brain in patients with alcohol addiction using VBM remains unclear. Since only few studies have focused on this issue, we aimed to systematically investigate the changes in brain structure in patients with alcohol addiction by analyzing the changes in gray and white matter volumes (WMVs) in patients with alcohol addiction using VBM with the hope of clarifying the underlying mechanism of alcohol-induced brain damage.

\section{Methods}

\section{Clinical data}

Twenty patients with alcohol addiction, consisting of 3 females and 17 males, aged 49 \pm 12 (range, 29-66) years, who received treatment between June 2017 to February 2018 in our hospital, were included in this study. For these patients, the length of education was $14 \pm 4$ (range, $0-19$ ) years and the body mass index was $25.8 \pm 2.9$ (range, $21.5-31.1$ ) $\mathrm{kg} / \mathrm{m}^{2}$. Age- and sex-matched healthy volunteers, consisting of 2

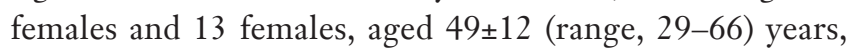
were included as controls. For these healthy controls, the length of education was $13 \pm 3$ (range, $8-16$ ) years and the body mass index was $23.1 \pm 1.0$ (range, $21.3-25.0$ ) $\mathrm{kg} / \mathrm{m}^{2}$. Independent samples $t$-test was used for comparisons of age, length of education, and body mass index between patients and healthy controls. There were no significant differences in these indices between patients with alcohol addiction and healthy controls (all $\mathrm{P}>0.05$ ). All included subjects were of Han nationality and were right-handed. Alcohol addiction was diagnosed according to a previously described guideline formulated by the World Health Organization (13). In addition, the included patients met the following criteria: (I) ethanol intake $\geq 30 \mathrm{~g} / \mathrm{d}$ over 10 years; (II) no history of hypertension, diabetes mellitus, or coronary heart disease; (III) no history of head trauma, cerebrovascular disease, or brain tumor; (IV) no history of smoking; (V) no history of addition of sedative drugs; (VI) no history of cirrhosis or alcoholic neurological dysfunction. All healthy controls underwent magnetic resonance imaging (MRI) scans of the whole-brain and no abnormal findings were observed. Written informed consent regarding the objective and methods of the study was obtained from each subject.

\section{Examination metbods}

A General Electric 3.0T HD Signa Excite scanner with an 8-channel head coil was used, with imaging gradients of a maximum strength of $40 \mathrm{mT} / \mathrm{m}$ and a slew rate of $150 \mathrm{mT} / \mathrm{m} / \mathrm{s}$. Fluid attenuated inversion recovery (FLAIR) sequence scans were performed in parallel to the anterior commissure (AC)-posterior commissure (PC) line with the following parameters: repetition time $(\mathrm{TR})=9,602.0 \mathrm{~ms}$, echo time $(\mathrm{TE})=108.0 \mathrm{~ms}$, inversion time $(\mathrm{TI})=2,400.0 \mathrm{~ms}$, layer thickness $=5 \mathrm{~mm}$, interval $=1 \mathrm{~mm}$, number of layers $=22$, field of view $(\mathrm{FOV})=24 \mathrm{~cm} \times 24 \mathrm{~cm}$, matrix $=320 \times 224$, and number of excitations $(\mathrm{NEX})=1$. Fast spoiled gradient echo (FSPGR) sequence scans were performed for the structure of the whole-brain including the scalp, with the following parameters: $\mathrm{TR}=6.3 \mathrm{~ms}, \mathrm{TE}=2.8 \mathrm{~ms}$, $\mathrm{TI}=450.0 \mathrm{~ms}$, layer thickness $=1 \mathrm{~mm}$, interval $=0 \mathrm{~mm}$, number of layers $=118$, $\mathrm{FOV}=24 \mathrm{~cm} \times 24 \mathrm{~cm}$, matrix $=256 \times 256$, and NEX $=1$. All MRI scans were performed by one experienced radiologist. During the MRI scans, the head of the subject was fixed with a sponge mat. MRI scans were performed at the anterior-posterior location along the midsagittal line, and the scanning range was from the scalp to the skull base.

\section{Data analysis and processing}

Raw data were statistically analyzed using Statistical Parametric Mapping 5 (SPM) software (http://www.fil.ion.ucl. ac.uk/spm/software/spm5) implemented in Matlab R2008a.

\section{Image registration}

All raw data were registered to the Montreal Neurological Institute (MNI) template using a 12-parameter affine registration. New images were then collected with a pixel of $1 \mathrm{~mm} \times 1 \mathrm{~mm} \times 1 \mathrm{~mm}$.

\section{Image segmentation}

The registered images were segmented as per GM, white matter, and cerebrospinal fluid (Figure 1A,B,C,D). According to Internal Consortium for Brain Mapping (ICBM), probability maps of the GM, white matter and cerebrospinal fluid were created. In the segmentation algorithm, a hidden Markov random field (HMRF) model (0.3) was selected. The voxel values in the segmented images were modulated as the volume of tissue in original images.

\section{Image smoothing}

An isotropic Gaussian kernel of $8 \mathrm{~mm}$ full-width at half 

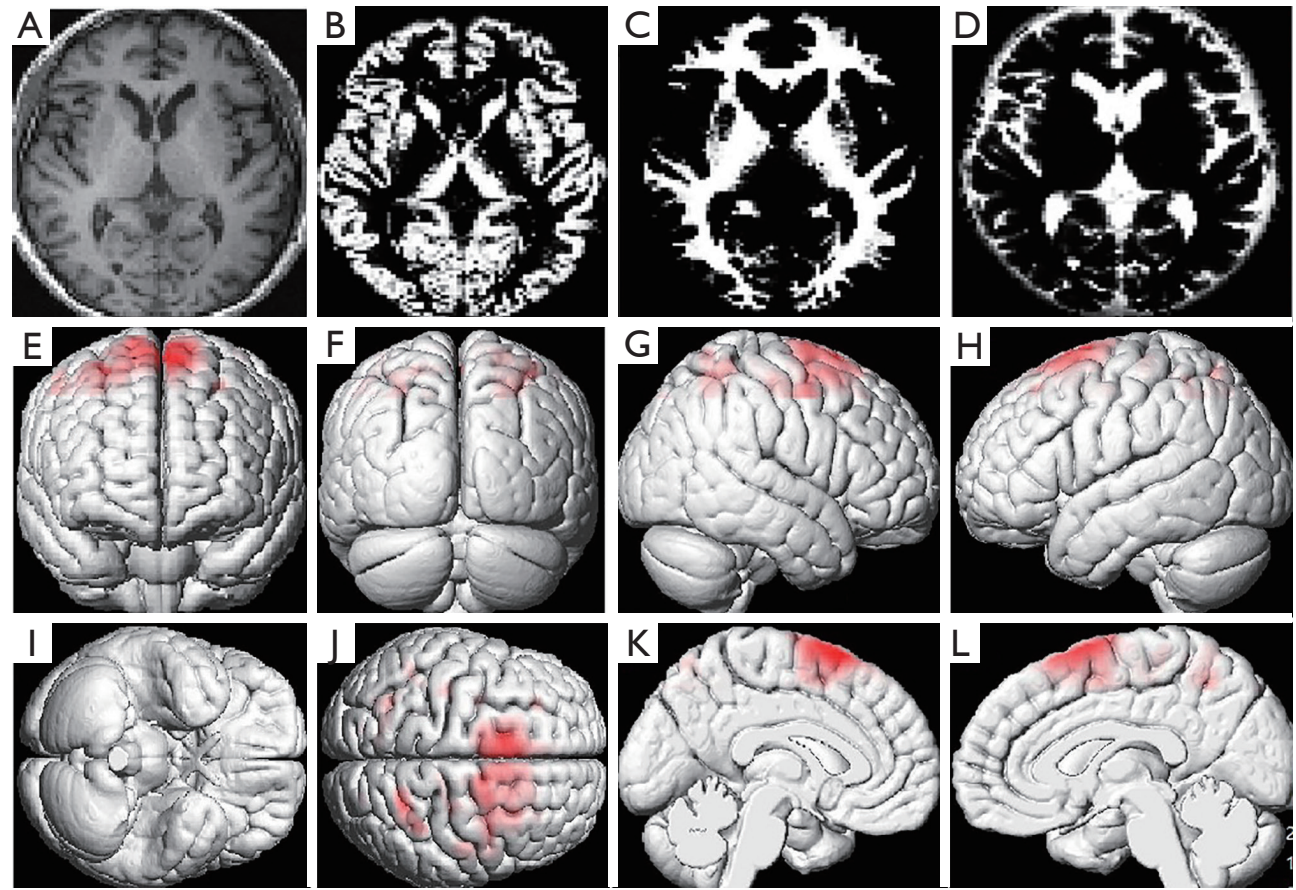

Figure 1 Probability maps of the whole-brain structure. (A) T1 structure image, (B) GM, (C) white matter, and (D) cerebrospinal fluid after image segmentation; reduced regional GMVs in patients with alcohol addiction compared to healthy controls apparent in the (E) left middle frontal gyrus, (F) left superior frontal gyrus, (G) left precuneus, (H) left gyrus rectus, (I) right medial superior frontal gyrus, and (J) right orbital gyrus; $(\mathrm{K}, \mathrm{L}) \mathrm{WMV}$ in the bilateral superior frontal gyri was also significantly reduced in patients with alcohol addiction compared to healthy controls. GM, gray matter; GMV, gray matter volume; WMV, white matter volume.

Table 1 The results of whole-brain volume analysis

\begin{tabular}{lcccc}
\hline Volume & $\begin{array}{c}\text { Alcohol addiction } \\
\text { group, } \mathrm{mL}\end{array}$ & Control group, $\mathrm{mL}$ & $t$ & $\mathrm{P}$ \\
\hline BPV & $1,584.34 \pm 139.56$ & $1,716.75 \pm 174.56$ & 2.649 & 0.011 \\
WMV & $823.57 \pm 73.77$ & $919.23 \pm 81.39$ & 3.894 & $<0.001$ \\
GMV & $912.45 \pm 138.32$ & $873.81 \pm 147.49$ & 0.854 & 0.398 \\
\hline
\end{tabular}

BPV, brain parenchyma volume; WMV, white matter volume; GMV, gray matter volume.

maximum (FWHM) was used to smooth the volumemodulated GM, white matter, and cerebrospinal fluid, with the aim of eliminating the errors generated during image reconstruction.

\section{Statistical analysis}

Statistical analysis was performed using SPSS (version 24.0; IBM Corporation, Armonk, NY, USA). Data are expressed as the mean \pm standard deviation. A two-sample $t$-test was used to compare quantitative data between the two groups, and a chi-square test was used for comparisons of qualitative data.

\section{Results}

\section{Whole-brain volume}

White matter and brain parenchyma volumes (BPVs) were significantly smaller in patients with alcohol addiction than in healthy controls (WMV: $823.57 \pm 73.77$ vs. $919.23 \pm 81.39 \mathrm{~mL}$; BPV: $1,584.34 \pm 139.56$ vs. $1,716.75 \pm 174.56 \mathrm{~mL}$; both $\mathrm{P}<0.05)$. There was no significant difference in GM volume (GMV) between patients with alcohol addiction and healthy controls $(912.45 \pm 138.32$ vs. $873.81 \pm 147.49 \mathrm{~mL} ; \mathrm{P}>0.05)$ (Table 1).

\section{Regional brain volumes}

GMV was significantly more reduced in the left middle frontal gyrus, left superior frontal gyrus, left precuneus, 
Table 2 Regional brain volumes reduced in patients with alcohol addiction compared to healthy controls

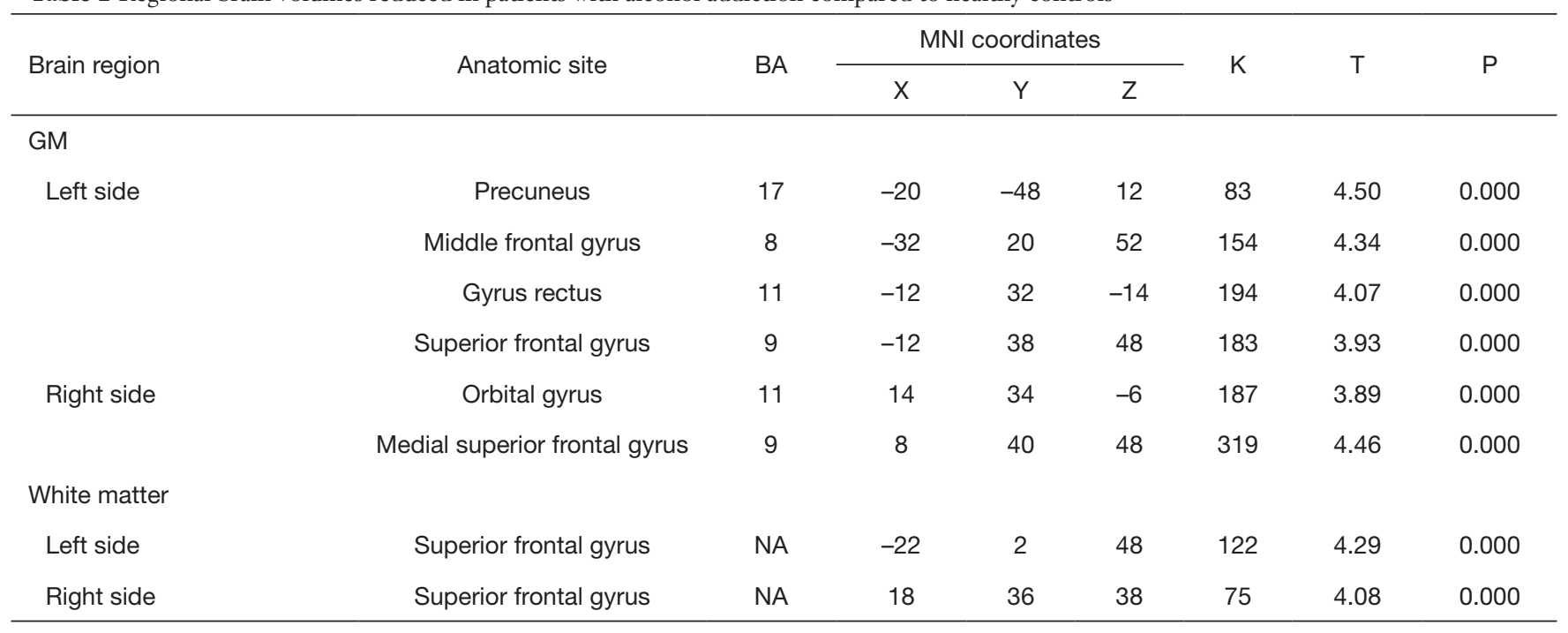

$\mathrm{X}, \mathrm{Y}, \mathrm{Z}$ are peak coordinates of activated voxel clusters given in $\mathrm{MNI}$ space; $\mathrm{K}$ is voxel value in the activated area; $\mathrm{T}$ is the area under the curve for analysis of activated area t. MNI, Montreal Neurological Institute; BA, Brodmann's area; GM, gray matter; NA, not available.

left gyrus rectus, right medial superior frontal gyrus, and right orbital gyrus in patients with alcohol addiction than in healthy controls (Table 2, Figure 1E,F,G,H,I, f). WMV in the bilateral superior frontal gyri was also significantly more reduced in patients with alcohol addiction than in healthy controls (Table 2, Figure $1 K, L$ ).

\section{Discussion}

\section{VBM results in patients with alcobol addiction}

A neuropathological study has demonstrated cortical atrophy, in particular frontal cortical atrophy, characterized by cell loss, destruction of cortical plate structure, and proliferation of glial components under the microscope, in patients with alcohol addiction. Frontal cortical atrophy occurs alone or together with other diseases, such as periventricular white matter damage and corpus callosum degeneration (14). However, a quantitative neuropathological study has demonstrated that white matter, rather than cerebral cortex, accounts for the majority of lost brain volume (15). Further quantitative analysis evidence exists showing that the number of neurons in the superior frontal gyrus and weights of brain tissue in patients with alcohol addiction were significantly smaller or lower than in healthy controls $(16,17)$. VBM findings regarding the changes in the whole-brain in patients with alcohol addiction make alcohol addiction-caused brain damage increasingly concerning, and providing scientific evidence for future imaging studies on this issue is necessary.

In this study, we used an advanced neuroimaging tool to analyze the structure of the whole-brain of patients with alcohol addiction. All patients included in this study had a history of alcohol abuse of more than 10 years. No abnormalities were observed on routine MR images, and all patients had no mental disorders. Thus, we ensured the obtained data were objective and accurate. VBM findings showed that white matter and BPVs were significantly smaller in patients with alcohol addiction than in healthy controls, but there was no significant difference in GMV between them, which is consistent with a previous report (18). Macroscopically, changes in brain tissue volumes are only to be used to roughly analyze the atrophy of brain volumes. To further analyze the features of atrophy in each brain region in patients with alcohol addiction and to find the specific brain regions for alcohol addiction-caused brain damage, we analyzed regional brain structure using VBM.

VBM can be used to objectively, sensitively, and repeatedly analyze the microscopic changes in a patient's brain region and can detect the early subtle changes in injured brain structure. Our results showed that the subtle atrophy of the GM in patients with alcohol addiction mainly occurred in the bilateral frontal lobes, in particular the superior frontal gyrus, but bilateral atrophic regions were not asymmetric, and the injured site of the white matter was located in the bilateral superior frontal gyrus. Our 
results, regarding alcohol addiction-caused brain damage, are to a certain degree consistent with previous pathological findings. Regarding this issue, imaging findings are superior to pathological outcomes because the former can be used to observe the subtle changes in brain structure in patients with alcohol addiction in vivo and provide more intuitive evidence for lesion detection and disease evaluation. Furthermore, MRI is preferred by patients because of its non-invasive nature.

\section{Changes in the frontal lobe of patients with alcohol addiction}

Our VBM quantitative analysis results showed that the specific brain regions (including GM and white matter) for alcohol addiction primarily include bilateral frontal lobes, in particular the superior frontal gyrus. Atrophy of the frontal lobe, particularly the superior frontal gyrus, is therefore considered as a biological marker of alcohol addictioncaused brain damage. Nevertheless, a study involving a larger sample size is needed to confirm this.

The frontal lobe is the largest cerebral cortical region that is composed of dorsal, medial, and basal orbital surfaces. It is a large association cortex, contains abundant afferent and efferent nerves, and is closely related to the structure of parietal lobe, occipital lobe, cingulate gyrus, limbic gyrus, and limbic gyrus (19). Therefore, the subtle atrophy of frontal lobe gray and white matter in patients with alcohol addiction is a leading cause of alcohol-related behavior problems. A previous study has demonstrated that severe chronic alcohol abuse can damage the neurons containing gamma-aminobutyric acid and benzodiazepine receptors on the medial surface of the frontal lobe, leading to hypometabolism of the frontal lobe. Nicolás et al. (20) reported that $65-68 \%$ of patients with alcohol addiction presented with decreased regional brain blood flow. MR spectroscopy findings (21) showed that the ratios of $\mathrm{N}$-acetylaspartate (NAA)/choline and NAA/creatine in the frontal lobe decreased in patients with long-term alcohol abuse. Decreased NAA level indicates loss of neurons in the frontal lobe, while decreased choline level suggests a marked change in cellular membrane lipids in patients with alcohol abuse (19). The present in vivo findings confirmed the subtle atrophy of GM and white matter in the bilateral frontal lobes in patients with alcohol addiction, and therefore it is presumed that the subtle change in frontal lobe structure likely contributes to the functional changes of the frontal lobe in patients with alcohol addiction. VBM quantitative analysis can provide powerful imaging evidence for evaluation of the subtle brain structure damage in patients with alcohol addiction.

The main objective of this study was to investigate the changes in cerebral structure in patients with alcohol addiction, and, given this focus, the change in cerebellar structure was not discussed. Since its clinical application in 1995, VBM has been widely used for the evaluation of brain structures in many diseases, but most of the studies have only evaluated the abnormal changes of cerebral structure. With further knowledge of cerebellar structure and functional regions and the development of cerebellar probability maps, detailed VBM analysis of cerebellar and brainstem structure can be realized.

VBM analysis can be used to objectively, automatically, quantitatively, and comprehensively evaluate the change in brain structure in patients with alcohol addiction, but voxel-based analysis possibly yields false positive results due to its hypersensitivity. Therefore, caution should be taken when performing VBM analysis. In addition, the selection of image sequence and mask threshold is also an influential factor of VBM results regarding atrophic brain regions. The present endpoints should be confirmed by pathological findings, which is a limitation to this study. In-depth longitudinal studies involving larger sample sizes should be performed. More accurate image registration and improvement in segmentation algorithms are necessary in future studies.

\section{Conclusions}

Taken together, the frontal lobe is a specific brain region for alcohol addiction-caused subtle atrophy of brain structure. VBM has certain value in evaluating cerebral atrophy in patients with alcohol addiction.

\section{Acknowledgments}

None.

\section{Footnote}

Conflicts of Interest: The authors have no conflicts of interest to declare.

Ethical Statement: The authors are accountable for all aspects of the work in ensuring that questions related to the accuracy or integrity of any part of the work are 
appropriately investigated and resolved. Written informed consent regarding the objective and methods of the study was obtained from each subject. The whole study procedure was approved and monitored by the Ethics Committee of PLA General Hospital, China.

\section{References}

1. Piras F, Piras F, Chiapponi C, et al. Widespread structural brain changes in OCD: a systematic review of voxel-based morphometry studies. Cortex 2015;62:89-108.

2. Hernández SE, Suero J, Barros A, et al. Increased grey matter associated with long-term Sahaja yoga meditation: a voxel-based morphometry study. PLoS One 2016;11:e0150757.

3. Nemoto K. Understanding voxel-based morphometry. Brain Nerve 2017;69:505-11.

4. Honea RA, Szabo-Reed AN, Lepping RJ, et al. Voxelbased morphometry reveals brain gray matter volume changes in successful dieters. Obesity (Silver Spring) 2016;24:1842-8.

5. Zhou Y, Lin FC, Du YS, et al. Gray matter abnormalities in Internet addiction: a voxel-based morphometry study. Eur J Radiol 2011;79:92-5.

6. Christidi F, Karavasilis E, Riederer F, et al. Gray matter and white matter changes in non-demented amyotrophic lateral sclerosis patients with or without cognitive impairment: a combined voxel-based morphometry and tract-based spatial statistics whole-brain analysis. Brain Imaging Behav 2018;12:547-63.

7. Wang S, Zhang R, Deng Y, et al. Discrimination of smoking status by MRI based on deep learning method. Quant Imaging Med Surg 2018;8:1113-20.

8. Chanson JB, Lamy J, Rousseau F, et al. White matter volume is decreased in the brain of patients with neuromyelitis optica. Eur J Neurol 2013;20:361-7.

9. Harper C. The neuropathology of alcohol-related brain damage. Alcohol Alcohol 2009;44:136-40.

10. Mechtcheriakov S, Brenneis C, Egger K, et al. A widespread distinct pattern of cerebral atrophy in patients with alcohol addiction revealed by voxelbased morphometry. J Neurol Neurosurg Psychiatry 2007;78:610-4.

11. Xiao P, Dai Z, Zhong J, et al. Regional gray matter deficits in alcohol dependence: a meta-analysis of voxel- based morphometry studies. Drug Alcohol Depend 2015;153:22-8.

12. Liu H, Hao Y, Kaneko Y, et al. Frontal and cingulate gray matter volume reduction in heroin dependence: optimized voxel-based morphometry. Psychiatry Clin Neurosci 2009;63:563-8.

13. Reed GM, Robles R, Domínguez-Martínez T. Classification of mental and behavioral disorders. In: Norcross JC, VandenBos GR, Freedheim DK, et al. editors. APA handbook of clinical psychology: Psychopathology and health. Washington: American Psychological Association, 2016.

14. Ridley NJ, Draper B, Withall A. Alcohol-related dementia: an update of the evidence. Alzheimers Res Ther 2013;5:3.

15. García-Valdecasas-Campelo E, González-Reimers E, Santolaria-Fernández F, et al. Brain atrophy in alcoholics: relationship with alcohol intake; liver disease; nutritional status, and inflammation. Alcohol Alcohol 2007;42:533-8.

16. Granhag PA, Rangmar J, Strömwall LA. Small cells of suspects: eliciting cues to deception by strategic interviewing. Journal of Investigative Psychology and Offender Profiling 2014. doi: 10.1002/jip.1413.

17. Gamito P, Oliveira J, Lopes P, et al. Assessment of frontal brain functions in alcoholics following a health mobile cognitive stimulation approach. Stud Health Technol Inform 2013;191:110-4.

18. Stewart JC, Rollman BL. Optimizing approaches to addressing depression in cardiac patients: a comment on O'Neil et al. Ann Behav Med 2014;48:142-4.

19. Badre D, Nee DE. Frontal cortex and the hierarchical control of behavior. Trends Cogn Sci 2018;22:170-88.

20. Nicolás JM, Estruch R, Salamero M, et al. Brain impairment in well-nourished chronic alcoholics is related to ethanol intake. Ann Neurol 1997;41:590-8.

21. Zahr NM, Sullivan EV, Rohlfing T, et al. Concomitants of alcoholism: differential effects of thiamine deficiency, liver damage, and food deprivation on the rat brain in vivo. Psychopharmacology (Berl) 2016;233:2675-86.

Cite this article as: $\mathrm{Li} \mathrm{J}$, Wang Y, Xu Z, Liu T, Zang X, Li M, $\mathrm{Ma} \mathrm{L}$. Whole-brain morphometric studies in alcohol addicts by voxel-based morphometry. Ann Transl Med 2019;7(22):635. doi: 10.21037/atm.2019.10.90 